\title{
Near-infrared images of star forming regions containing masers
}

\section{Las Campanas observations of 31 southern sources $^{\star, \star \star}$}

L. Testi ${ }^{1,2}$, M. Felli ${ }^{3}$, P. Persi ${ }^{4}$, and M. Roth $^{5}$

1 Division of Physics, Mathematics and Astronomy, California Institute of Technology, MS105-24, Pasadena, CA 91125, U.S.A.

2 Dipartimento di Astronomia e Scienza dello Spazio, Università degli Studi di Firenze, Largo E. Fermi 5, I-50125 Firenze, Italy

3 Osservatorio Astrofisico di Arcetri, Largo E. Fermi 5, I-50125 Firenze, Italy

4 Istituto di Astrofisica Spaziale, C.N.R., C.P. 67, I-00044 Frascati, Italy

${ }^{5}$ Las Campanas Observatory, Casilla 601, La Serena, Chile

Received July 17; accepted October 24, 1997

\begin{abstract}
We present sensitive high resolution near infrared (NIR) broad band $(J, H$, and $K)$ observations of a sample of 31 Star Forming Regions (SFRs) which contain $\mathrm{H}_{2} \mathrm{O}$ and $\mathrm{OH}$ maser sources.

The observations are aimed at the detection and characterization of Young Stellar Objects (YSOs) which may be the source of excitation of the maser emission. In spite of the large number of sources detected in the regions, using positional coincidence and NIR colours we are able to reliably identify $K$-band sources related to the masing gas in a large fraction of the observed regions.

The NIR infrared sources selected from close positional coincidence with the maser show strong NIR excesses and most probably represent the YSOs still embedded in their parental cocoon where the maser emission occurs.
\end{abstract}

Key words: masers - star formation - circumstellar matter — infrared: ISM: continuum; stars

\section{Introduction}

$\mathrm{H}_{2} \mathrm{O}$ masers were found in SFRs since the earliest spectral line surveys (Genzel \& Downes 1977) and were recognized to be closely related with young massive stars. More recently Palla et al. (1993) and Codella et al. (1994) suggested that water maser activity is indeed present in the earliest evolutionary phases of a high mass (proto-)star,

\footnotetext{
Send offprint requests to: Testi: Caltech, lt@astro.caltech.edu * Based on observations collected at Las Campanas Observatory, Carnegie Institution of Washington.

$\star \star$ Figure 1 is can be accessed on the online version of the journal (http://www.edpsciences.com).
}

even before the onset of an UCHII region. Nevertheless, it was clear that the comparison of the low resolution data (e.g. single dish maser observations, radio continuum observations of the free-free emission from HII regions and IRAS observations of cool dust clouds around luminous stars) could give but a general indication. Only higher resolution $\left(\lesssim 1^{\prime \prime}\right)$ observations can enable one to disentangle the complexity of high mass SFRs and allow one to search for the stellar source directly connected to the maser. In fact, according to the shock excitation model of Elitzur et al. (1989), the exciting stellar source should be located very close to the maser $\left(\leq 10^{4} \mathrm{AU}\right.$ or $\leq 10^{\prime \prime}$ at $1 \mathrm{kpc})$. Consequently, arcmin coincidences have very little significance.

On the radio side of the spectrum the NRAO Very Large Array (VLA) offers the possibility of obtaining subarcsec resolutions for the water masers and for the radio continuum emission from UCHII regions (Forster \& Caswell 1989, hereafter FC89; Tofani et al. 1995; Jenness et al. 1995; Hofner \& Churchwell 1996). One of the most important results of these studies has been that $\mathrm{H}_{2} \mathrm{O}$ masers and UCHII regions, although generally found in the same SFR, are not necessarely closely related to each other, and that the powering sources of the free-free continuum and of the maser radiation have to be searched in distinct objects, most probably YSOs belonging to the same SFR but in different evolutionary phases.

Consequently, to identify the stellar source powering the maser, arcsecond resolution observations are required, in particular in the far infrared and submillimeter regions of the spectrum. In fact, it is in these wavebands that the embedded YSOs are expected to emit most of their energy. Although some attempts in this direction have been carried out (Jenness et al. 1995), the current 
instrumentation does not allow one to attain the required resolution and sensitivity in these spectral ranges.

Another powerful probe that became available in the past few years is molecular line interferometry at centimeter and millimeter wavelengths (Cesaroni et al. 1994; Cesaroni et al. 1997; Codella et al. 1997; Hofner et al. 1996; Olmi et al. 1996) which enables arcsecond resolution of hot molecular clumps and cold dust clouds surrounding the water masers. These studies, focused on a few selected sources, confirm that the water masers are indeed associated with hot molecular clumps and millimeter continuum sources, and that the expected luminosities of the objects embedded inside these cores is that of high mass stars. However, this method is not feasible for large surveys.

In order to search for the stellar sources physically associated with the masers we decided to undertake a systematic NIR imaging survey of a sample of SFRs containing masers. This is motivated by the fact that the modern NIR array detectors offer the required sensitivity and resolution even with small telescopes and with short integration times. We were also guided by the expectation that in its early evolutionary stages high mass stars could be detectable at NIR wavelengths even if still deeply embedded in a dust cloud, due to the emission of hot dust around the star (Testi et al. 1997). Previous attempts to look for near infrared sources close to $\mathrm{H}_{2} \mathrm{O}$ masers had been carried out with single element photometers and large diaphragms (Evans et al. 1979; Moorwood \& Salinari 1981a,b; Epchtein \& Lépine 1981; Braz \& Epchtein 1982) and could not reach the sensitivities and resolutions needed to separate the various YSOs in each region.

In this paper we present NIR observations with arcsec resolution of a sample of $\mathrm{H}_{2} \mathrm{O} / \mathrm{OH}$ masers in SFRs taken from the list of FC89. The complete radio data were kindly provided by R. Forster (1992 private communication).

The SFR type of the masers in our sample (to distinguish them from those around late type stars) relies on the selection criteria used by FC89, basically the presence of a thermal continum radio source (an HII region) close to the maser. As an additional check, we have derived for those fields that contain an IRAS point source $(27$ out of $31)$ the $(25 \mu \mathrm{m} / 12 \mu \mathrm{m})$ and the $(60 \mu \mathrm{m} / 12 \mu \mathrm{m})$ colours. All the points fall in the box of the $(25 \mu \mathrm{m} / 12 \mu \mathrm{m})-$ $(60 \mu \mathrm{m} / 12 \mu \mathrm{m})$ diagram that defines the locus of UCHII regions (Wood \& Churchwell 1989). This is a further confirmation of the star forming nature of the selected fields, even though, strictly speaking, it is not directly related to the nature of the star associated with the maser itself.

In Testi et al. (1994) (hereafter Paper I) we have presented the results obtained toward a subsample of 17 SFRs, which is in fact the first attempt to compare maser and NIR observations at arcsec resolution. Assuming that a NIR source is physically associated with the maser if it lies within $10^{\prime \prime}$ from it, we were able to identify a NIR source in $\sim 85 \%$ of the cases. In $\sim 80 \%$ the NIR source was closer than $5^{\prime \prime}$. In this paper we present new higher resolution and higher sensitivity observations of 31 SFRs from the list of FC89 (5 regions overlap with that presented in Paper I and are indicated with an $*$ in Table 1). Three of the regions in the present sample (G351.41+0.64; G009.62+0.19; G035.20-1.73) have been studied also with high resolution observations at other frequencies (Persi et al. 1996; Testi et al. 1997 and Persi et al. 1997). In all these cases the physical association of the selected NIR source with the maser is amply confirmed by independent criteria. In Sect. 2 the observational parameters and the data reduction procedures are summarized. In Sect. 3 the results are shown. In Sect. 4 we show that close positional coincidence alone is not sufficient to establish a physical association, and that the NIR colour is the complementary information. In fact, the NIR sources associated with the masers all show a strong NIR excess, while the background NIR sources are distributed along the reddening line. In Sect. 5 the main conclusions are summarized.

\section{Observations}

The observed $\mathrm{H}_{2} \mathrm{O}$ and $\mathrm{OH}$ masers are listed in Table 1. For each source we report the FC89 name (the 5 presented in Paper I and reobserved in June 1994 are marked with an $*$ ), the 1950 coordinates of the $\mathrm{H}_{2} \mathrm{O}$ maser and the distance from the Sun (from FC89), the IRAS name of the IRAS-PSC source (if any within 1 arcmin from the maser position), and the limiting magnitudes achieved in our observations (see below). All the sources were observed in $J, H$ and $K(1.25 \mu \mathrm{m}, 1.65 \mu \mathrm{m}$ and $2.2 \mu \mathrm{m}$ respectively) NIR broad bands using the Las Campanas NIR camera (Persson et al. 1992) equipped with a NICMOS3 $256 \times 256 \mathrm{HgCdTe}$ array detector. The sources were observed in two runs at the $1 \mathrm{~m}$ telescope (July 1992) and at the $2.5 \mathrm{~m}$ telescope (June 1994). The observational and data reduction techniques were the same as for the preliminary sample presented in Paper I. The plate scale on the detector was $0.92^{\prime \prime} /$ pix at the $1 \mathrm{~m}$ telescope and $0.35^{\prime \prime} /$ pix at the $2.5 \mathrm{~m}$. During both observing runs the seeing was in the range $0.8^{\prime \prime}-1.5^{\prime \prime}$. The data reduction and analysis were performed using the IRAF $^{1}$ and $\mathrm{ARNICA}^{2}$ (Hunt et al. 1994) software packages.

Photometric calibration was achieved by observing a set of the UKIRT faint standard stars (Casali \& Hawardeen 1992) each night. The calibration accuracy is in the range $5 \%-10 \%$ depending on the weather conditions. The limiting magnitudes obtained in each field ( $3 \sigma$ in a 4 pix aperture) are listed in Table 1 . Photometry was performed using the IRAF DAOPHOT package.

1 IRAF is made available to the astronomical community by the National Optical Astronomy Observatories, which are operated by AURA, Inc., under contract with the U.S. National Science Foundation.

2 ARNICA can be obtained from the Osservatorio Astrofisico di Arcetri at ftp://150.217.20.1/pub/arnica/ 
Table 1. Parameters of the observed regions

\begin{tabular}{|c|c|c|c|c|c|c|c|c|}
\hline & Name & $\alpha(1950)$ & $\delta(1950)$ & $D(\mathrm{kpc})$ & IRAS & $m l_{J}$ & $m l_{H}$ & $m l_{K}$ \\
\hline \multicolumn{9}{|c|}{ Las Campanas $1 \mathrm{~m}$ telescope, July 1992} \\
\hline & G351.41+0.64 & $17: 17: 32.34$ & $-35: 44: 02.5$ & 1.9 & $17175-3544$ & 16.5 & 15.8 & 14.6 \\
\hline & G351.78-0.54 & $17: 23: 20.32$ & $-36: 06: 44.0$ & 2.2 & $17233-3606$ & 16.6 & 16.1 & 15.3 \\
\hline & G353.41-0.36 & $17: 27: 05.56$ & $-34: 39: 21.7$ & 4.5 & $17271-3439$ & 16.5 & 15.9 & 15.0 \\
\hline & G355.34+0.15 & $17: 30: 12.54$ & $-32: 45: 56.6$ & 2.0 & $17302-3245$ & 16.3 & 15.2 & 14.3 \\
\hline & G359.62-0.25 & $17: 42: 27.70$ & $-29: 22: 21.2$ & 10.0 & - & 16.5 & 15.1 & 14.2 \\
\hline & G005.88-0.39 & $17: 57: 26.83$ & $-24: 03: 56.5$ & 2.6 & $17574-2403$ & 16.5 & 15.5 & 14.5 \\
\hline & G009.62+0.19 & 18:03:15.99 & $-20: 31: 54.9$ & 2.0 & $18032-2032$ & 16.7 & 15.4 & 14.5 \\
\hline & G011.03+0.06 & $18: 06: 42.56$ & $-19: 21: 56.0$ & 3.2 & $18067-1921$ & 16.0 & 15.0 & 14.5 \\
\hline & G010.62-0.38 & $18: 07: 30.56$ & $-19: 56: 28.8$ & 6.0 & $18075-1956$ & 16.5 & 15.7 & 14.8 \\
\hline & G012.22-0.12 & 18:09:43.81 & $-18: 25: 05.7$ & 16.1 & $18097-1825$ & 16.1 & 15.0 & 14.4 \\
\hline & G015.04-0.68 & $18: 17: 29.91$ & $-16: 13: 11.0$ & 2.5 & $18174-1612$ & 16.0 & 15.1 & 15.0 \\
\hline & G019.61-0.23 & $18: 24: 50.25$ & $-11: 58: 31.7$ & 3.8 & $18248-1158$ & 15.5 & 14.5 & 14.5 \\
\hline & G023.43-0.19 & $18: 31: 55.53$ & $-08: 34: 03.9$ & 7.8 & - & 16.0 & 15.1 & 15.0 \\
\hline & G023.01-0.41 & $18: 31: 56.10$ & $-09: 03: 03.7$ & 12.8 & - & 16.3 & 15.3 & 14.7 \\
\hline & G024.78+0.08 & 18:33:30.43 & $-07: 14: 43.2$ & 7.7 & $18335-0713 \mathrm{~A}$ & 16.0 & 15.1 & 15.0 \\
\hline \multicolumn{9}{|c|}{ Las Campanas $2.5 \mathrm{~m}$ telescope, June 1994} \\
\hline \multirow[t]{2}{*}{ * } & G345.01+1.79 & $16: 53: 17.28$ & $-40: 09: 23.2$ & 3.0 & $16533-4009$ & 19.3 & 18.3 & 17.0 \\
\hline & G343.12-0.06 & $16: 54: 44.03$ & $-42: 47: 36.6$ & 3.2 & $16547-4247$ & 19.2 & 18.2 & 17.2 \\
\hline * & G345.51+0.35 & $17: 00: 53.55$ & $-40: 40: 18.5$ & 2.1 & $17008-4040$ & 19.0 & 17.4 & 17.1 \\
\hline$*$ & G345.00-0.22 & $17: 01: 40.14$ & $-41: 24: 58.5$ & 3.2 & $17016-4124$ & 19.5 & 18.5 & 17.2 \\
\hline \multirow[t]{11}{*}{ * } & G345.40-0.90 & $17: 06: 02.18$ & $-41: 32: 06.3$ & 2.3 & $17059-4132$ & 17.5 & 16.0 & 15.5 \\
\hline & G349.07-0.01 & $17: 13: 25.55$ & $-38: 01: 58.8$ & 1.0 & $17134-3802$ & 18.1 & 16.5 & 16.4 \\
\hline & G350.02+0.43 & $17: 14: 22.13$ & $-37: 00: 03.2$ & 5.0 & $17143-3700$ & 18.9 & 17.1 & 16.9 \\
\hline & G348.73-1.06 & $17: 16: 39.83$ & $-38: 54: 13.6$ & 1.8 & $17167-3854$ & 19.0 & 17.3 & 16.2 \\
\hline & G351.58-0.35 & $17: 22: 03.27$ & $-36: 10: 05.5$ & 9.9 & $17220-3609$ & 19.0 & 17.2 & 17.0 \\
\hline & G353.45+0.54 & $17: 23: 33.02$ & $-34: 05: 54.6$ & 8.4 & - & 19.5 & 17.0 & 16.8 \\
\hline & G352.51-0.15 & $17: 23: 50.87$ & $-35: 17: 00.9$ & 8.0 & $17238-3516$ & 19.3 & 17.4 & 16.7 \\
\hline & G354.61+0.48 & $17: 26: 59.99$ & $-33: 11: 38.2$ & 4.2 & $17269-3312$ & 19.0 & 17.2 & 16.8 \\
\hline & G359.14+0.03 & $17: 40: 13.96$ & $-29: 37: 57.9$ & 5.0 & $17402-2938$ & 19.2 & 16.9 & 15.4 \\
\hline & G359.97-0.46 & $17: 44: 09.22$ & $-29: 10: 57.7$ & 10.0 & $17441-2910$ & 18.3 & 16.8 & 15.6 \\
\hline & G002.14+0.01 & $17: 47: 28.19$ & $-27: 04: 59.2$ & 8.9 & $17474-2704$ & 18.6 & 16.5 & 16.2 \\
\hline * & G035.20-1.73 & $18: 59: 13.24$ & $+01: 09: 13.5$ & 2.9 & $18592+0108$ & 19.6 & 17.6 & 17.0 \\
\hline
\end{tabular}

The key aspect of this project is the comparison of the NIR images with sub-arcsec resolution positions of the masers. Consequently, it is important to perform an accurate astrometric calibration of the NIR images. This was performed following the procedure outlined in Testi 1993 using the Digitized Sky Survey provided by the Space Telescope Science Institute. We reached an accuracy better than $1^{\prime \prime}$ rms.

\section{Results}

In Figs. 1a-p the $J, H$ and $K$ images for all the observed fields are presented. The fields are presented in the same order as in Table 1. The greyscale display is logarithmic in all cases. The crosses and the open squares represent the positions of the $\mathrm{H}_{2} \mathrm{O}$ and $\mathrm{OH}$ maser spots detected by FC89. The circles represent the peak positions of the UCHII regions, when detected by FC89 (however, note that their continuum observations are not sensitive to sources weaker than $\sim 150 \mathrm{mJy}$ at $22 \mathrm{GHz}$ ). The large ellipse represents the IRAS-PSC error box (note that due to an error in the plotting routine the ellipse for G035.20-1.73 in Fig. 2 of Paper I was plotted west of north instead of east of north). For each NIR image the 1950 coordinate grid was derived from the astrometry.

\subsection{Maser morphology}

Following the reasoning of Paper I, in order to compare the radio data with the NIR images we have grouped the $\mathrm{H}_{2} \mathrm{O}$ and $\mathrm{OH}$ maser spots detected by FC89 in each field into components of $\sim 1^{\prime \prime}$ size. In this way, in the 31 fields we find a total of $68 \mathrm{H}_{2} \mathrm{O}$ and $40 \mathrm{OH}$ maser components. The components have been identified following the convention of Paper I.

\subsection{Close coincidences between NIR sources and masers}

The necessary (but not sufficient) requirement to establish a physical association between a maser and a NIR source 
Fig. 1. a-p) $J, H$ and $K$ images of all the observed regions. The greyscale is logarithmic in all cases, crosses and open squares represent the $\mathrm{H}_{2} \mathrm{O}$ and $\mathrm{OH}$ maser spots detected by FC89, respectively. Open circles mark the position of the radio continuum peak (where detected by FC89). The large ellipses represent the IRAS-PSC error boxes. The coordinate grid, derived from the astrometry, are at 1950 (available in electronic form)

is close positional coincidence. For each maser we have searched for the closest source in the $K$-band. The NIR source was considered to be physically associated with the maser component if closer than $5^{\prime \prime}$ (see below). We decided to use the nearest neighbour approach in the association in order to avoid any a priori bias on the associated sources (see the discussion in Paper I).

In Tables $2 \mathrm{a}$ and $2 \mathrm{~b}$ the parameters are listed for the NIR sources associated with the maser components. For each NIR source we list an identifyer derived from the galactic coordinates (e.g. g35141-1 for the NIR source associated with the first maser component in G351.41+0.64), the 1950 coordinates, the magnitudes with errors in the $J, H$ and $K$ bands, the maser component with which it is associated, the separation between the maser component and the NIR source in arcseconds and in milliparsecs (i.e. projected on the plane of the sky using the distance reported by FC89). When a source is not detected in one or more wavebands the limiting magnitude is indicated and the lower limit is identified with a ">" symbol. When a maser group was not coincident with any NIR sources, the NIR identifyer is marked with an "N" and the limiting magnitudes in the three bands are given.

It is worth noting that all the maser components in the 1994 sample have a NIR source within $5^{\prime \prime}$. This is primarly due to the much higher sensitivity of the observations with respect to those of the 1992 run and especially those presented in Paper I. For this reason the possibility of spurious coincidence should be reconsidered in view of the higher quality of the data available (see Sect. 4). Anticipating the results of the following section, we decided to retain as good physical associations the coincidences within $5^{\prime \prime}$ and mark as dubious all the coincidences at larger distances (marked with "D" in Table 2). Extremely crowded regions in which the probability of spurious coincidences is very high have been indicated in Table $2 \mathrm{~b}$ with a "C" in the last column.

For the following discussion we will merge the data presented in this paper with that of Paper I. Due to the better resolution and sensitivity, for the 5 sources of Paper I that have been reobserved in June 1994 we will consider only the observations presented here. Consequently, the total number of SFRs is 43 and the numbers of $\mathrm{H}_{2} \mathrm{O}$ and $\mathrm{OH}$ masers are 82 and 53, respectively. In Fig. 2 we show the distribution of the separations between the first $K$-band neighbour and the $\mathrm{OH}$ and $\mathrm{H}_{2} \mathrm{O}$ maser components, in arcseconds and in physical separation projected on the plane of the sky (assuming the distance quoted in Table 1). Note that in the figure we have considered also the association with $5^{\prime \prime}<d<10^{\prime \prime}$, to be consistent with Paper I. In the following section we will discuss the reliability of such associations.

In Fig. 3 we show the difference in separation of the $\mathrm{H}_{2} \mathrm{O}$ and $\mathrm{OH}$ maser components associated with the same NIR source. The histogram is strongly peaked around zero and does not show any significant asymmetry, indicating that there does not seem to be any trend for one of the two maser types to form closer to the associated NIR source.

\subsection{Comparison between the 1991 and 1994 observations}

Five of the sources have been observed both in 1991 at the $1 \mathrm{~m}$ telescope with the NICMOS2-based camera (Paper I) and in 1994 at the $2.5 \mathrm{~m}$ telescope with the new detector (this paper).

In several cases (sources: g34500-1; g34501-1,2,3,4; g34541-1,2; g34551-1) the new higher resolution and higher sensitivity observations enabled detection of new sources (closer to the maser components than those reported in Paper I) fainter than the old detection limit or confused because they are embedded in diffuse nebulositities, which prevented a reliable detection in the lower resolution observations. In one case (g34500-2) the pointlike source is surrounded by a diffuse halo (unresolved in the 1991 observations) and hence the integrated magnitude (that of Paper I) is 1 magnitude brighter than that reported in Table 2. The other two sources (g03520-1 and g34551-2) are in excellent agreement with that of Paper I, taking into account the photometric calibration uncertainties.

We thus conclude that in a few cases the new observations found a NIR source associated with the maser component where no sources were found in Paper I and in other cases the agreement between the two sets of observations is rather good. However, in some cases the source associated with the maser components in Paper I is not the closest to the maser when higher sensitivity data are considered. This fact implies that (especially for the associations with larger separation) the confusion due to unrelated background sources should be carefully considered.

\section{Nature of the NIR sources}

Due to the higher data quality the sources surface density at $K$-band near the maser positions in the 1994 observations is a factor $3-20$ (depending on the field) higher than in the 1991 observations (see Paper I). As a consequence, for all the maser components we can find a NIR source 
Table 2. a) NIR sources associated with the maser emission, 1992 observations

\begin{tabular}{|c|c|c|c|c|c|c|c|c|c|}
\hline Name & $\alpha(1950)$ & $\delta(1950)$ & $m_{J}$ & $m_{H}$ & $m_{K}$ & Maser & $d\left({ }^{\prime \prime}\right)$ & $d(\mathrm{mpc})$ & \\
\hline \multirow[t]{3}{*}{ g35141-1 } & $17: 17: 32.26$ & $-35: 44: 04.6$ & $15.80 \pm 0.18$ & $13.30 \pm 0.04$ & $10.02 \pm 0.01$ & $\mathrm{H}_{2} \mathrm{O}-\mathrm{A}$ & 2.3 & 21.2 & \\
\hline & & & & & & $\mathrm{H}_{2} \mathrm{O}-\mathrm{B}$ & 7.4 & 68.2 & $\mathrm{D}$ \\
\hline & & & & & & OH-A & 2.7 & 24.9 & \\
\hline \multirow[t]{6}{*}{ g35178-1 } & $17: 23: 20.49$ & $-36: 06: 40.2$ & $13.60 \pm 0.02$ & $12.98 \pm 0.02$ & $11.73 \pm 0.02$ & $\mathrm{H}_{2} \mathrm{O}-\mathrm{A}$ & 4.3 & 45.9 & \\
\hline & & & & & & $\mathrm{H}_{2} \mathrm{O}-\mathrm{B}$ & 2.4 & 25.6 & \\
\hline & & & & & & $\mathrm{H}_{2} \mathrm{O}-\mathrm{C}$ & 3.2 & 34.1 & \\
\hline & & & & & & $\mathrm{H}_{2} \mathrm{O}-\mathrm{D}$ & 9.9 & 105.6 & $\mathrm{D}$ \\
\hline & & & & & & OH-A & 6.7 & 71.5 & $\mathrm{D}$ \\
\hline & & & & & & OH-B & 5.0 & 53.3 & \\
\hline \multirow{2}{*}{ g35341-N } & & & $>16.5$ & $>15.9$ & $>15.0$ & $\mathrm{H}_{2} \mathrm{O}-\mathrm{A}$ & - & - & \\
\hline & & & & & & $\mathrm{OH}-\mathrm{A}$ & - & - & \\
\hline g35341-1 & $17: 27: 08.94$ & $-34: 39: 33.9$ & $15.00 \pm 0.08$ & $14.11 \pm 0.07$ & $11.90 \pm 0.06$ & $\mathrm{H}_{2} \mathrm{O}-\mathrm{B}$ & 1.7 & 37.1 & \\
\hline g35534-1 & $17: 30: 12.57$ & $-32: 45: 56.2$ & $16.10 \pm 0.23$ & $14.48 \pm 0.16$ & $13.52 \pm 0.20$ & $\mathrm{H}_{2} \mathrm{O}-\mathrm{A}$ & 0.6 & 5.8 & \\
\hline \multirow[t]{2}{*}{ g35534-2 } & $17: 30: 12.39$ & $-32: 45: 41.8$ & $15.30 \pm 0.10$ & $12.23 \pm 0.02$ & $10.67 \pm 0.01$ & $\mathrm{H}_{2} \mathrm{O}-\mathrm{B}$ & 5.3 & 51.4 & $\mathrm{D}$ \\
\hline & & & & & & OH-A & 0.6 & 5.8 & \\
\hline \multirow[t]{2}{*}{ g35962-1 } & $17: 42: 28.07$ & $-29: 22: 21.5$ & $14.40 \pm 0.05$ & $11.56 \pm 0.01$ & $9.20 \pm 0.01$ & $\mathrm{H}_{2} \mathrm{O}-\mathrm{A}$ & 4.8 & 232.7 & \\
\hline & & & & & & $\mathrm{H}_{2} \mathrm{O}-\mathrm{B}$ & 9.3 & 450.9 & $\mathrm{D}$ \\
\hline \multirow[t]{8}{*}{ g00588-1 } & $17: 57: 26.91$ & $-24: 03: 58.1$ & $16.00 \pm 0.29$ & $13.03 \pm 0.05$ & $10.19 \pm 0.01$ & $\mathrm{H}_{2} \mathrm{O}-\mathrm{A}$ & 3.4 & 42.9 & \\
\hline & & & & & & $\mathrm{H}_{2} \mathrm{O}-\mathrm{B}$ & 2.2 & 27.7 & \\
\hline & & & & & & $\mathrm{H}_{2} \mathrm{O}-\mathrm{C}$ & 2.4 & 30.3 & \\
\hline & & & & & & $\mathrm{H}_{2} \mathrm{O}-\mathrm{D}$ & 3.3 & 41.6 & \\
\hline & & & & & & $\mathrm{H}_{2} \mathrm{O}-\mathrm{E}$ & 6.9 & 87.0 & $\mathrm{D}$ \\
\hline & & & & & & $\mathrm{OH}-\mathrm{A}$ & 5.7 & 71.8 & $\mathrm{D}$ \\
\hline & & & & & & $\mathrm{OH}-\mathrm{B}$ & 1.1 & 13.9 & \\
\hline & & & & & & $\mathrm{OH}-\mathrm{C}$ & 4.8 & 60.5 & \\
\hline g00588-N & & & $>16.5$ & $>15.5$ & $>14.5$ & $\mathrm{H}_{2} \mathrm{O}-\mathrm{F}$ & - & - & \\
\hline \multirow[t]{4}{*}{ g00962-2 } & $18: 03: 15.94$ & $-20: 31: 53.6$ & $15.50 \pm 0.07$ & $15.00 \pm 0.20$ & $14.00 \pm 0.20$ & $\mathrm{H}_{2} \mathrm{O}-\mathrm{A}$ & 1.4 & 13.6 & \\
\hline & & & & & & $\mathrm{H}_{2} \mathrm{O}-\mathrm{B}$ & 1.9 & 18.4 & \\
\hline & & & & & & OH-A & 1.5 & 14.5 & \\
\hline & & & & & & OH-B & 4.2 & 40.7 & \\
\hline \multirow[t]{3}{*}{ g00962-1 } & $18: 03: 16.15$ & $-20: 32: 00.0$ & $>16.4$ & $>15.4$ & $12.90 \pm 0.08$ & $\mathrm{H}_{2} \mathrm{O}-\mathrm{C}$ & 0.7 & 6.8 & \\
\hline & & & & & & $\mathrm{H}_{2} \mathrm{O}-\mathrm{D}$ & 2.7 & 26.2 & \\
\hline & & & & & & OH-C & 3.7 & 35.9 & \\
\hline \multirow[t]{2}{*}{ g01103-1 } & $18: 06: 42.69$ & $-19: 21: 55.5$ & $14.90 \pm 0.10$ & $13.34 \pm 0.07$ & $12.30 \pm 0.07$ & $\mathrm{H}_{2} \mathrm{O}-\mathrm{A}$ & 2.1 & 32.6 & \\
\hline & & & & & & OH-A & 1.8 & 27.9 & \\
\hline \multirow[t]{5}{*}{ g01062-2 } & $18: 07: 30.90$ & $-19: 56: 28.1$ & $16.50 \pm 0.24$ & $15.08 \pm 0.10$ & $13.50 \pm 0.09$ & $\mathrm{H}_{2} \mathrm{O}-\mathrm{A}$ & 4.9 & 142.5 & \\
\hline & & & & & & $\mathrm{H}_{2} \mathrm{O}-\mathrm{B}$ & 6.4 & 186.2 & $\mathrm{D}$ \\
\hline & & & & & & $\mathrm{H}_{2} \mathrm{O}-\mathrm{C}$ & 8.5 & 247.3 & $\mathrm{D}$ \\
\hline & & & & & & OH-A & 3.3 & 96.0 & \\
\hline & & & & & & $\mathrm{OH}-\mathrm{B}$ & 1.4 & 40.7 & \\
\hline g01062-1 & $18: 07: 31.24$ & $-19: 56: 28.3$ & $15.30 \pm 0.08$ & $14.38 \pm 0.06$ & $14.25 \pm 0.18$ & $\mathrm{H}_{2} \mathrm{O}-\mathrm{D}$ & 2.5 & 72.7 & \\
\hline \multirow[t]{2}{*}{ g01222-2 } & $18: 09: 43.94$ & $-18: 25: 07.8$ & $13.70 \pm 0.03$ & $11.61 \pm 0.02$ & $10.75 \pm 0.02$ & $\mathrm{H}_{2} \mathrm{O}-\mathrm{A}$ & 5.5 & 429.3 & $\mathrm{D}$ \\
\hline & & & & & & $\mathrm{H}_{2} \mathrm{O}-\mathrm{B}$ & 3.8 & 296.6 & \\
\hline \multirow[t]{2}{*}{ g01222-1 } & $18: 09: 48.74$ & $-18: 25: 15.0$ & $14.20 \pm 0.05$ & $12.66 \pm 0.03$ & $12.34 \pm 0.06$ & $\mathrm{H}_{2} \mathrm{O}-\mathrm{C}$ & 4.0 & 312.2 & \\
\hline & & & & & & OH-A & 4.2 & 327.8 & \\
\hline g01504-1 & $18: 17: 29.88$ & $-16: 13: 15.8$ & $13.80 \pm 0.09$ & $12.59 \pm 0.06$ & $12.03 \pm 0.10$ & $\mathrm{H}_{2} \mathrm{O}-\mathrm{A}$ & 4.8 & 58.2 & \\
\hline g01504-N & & & $>160$ & $>15.1$ & $>15.0$ & $\mathrm{H}_{2} \mathrm{O}-\mathrm{B}$ & - & - & \\
\hline g01504-2 & $18: 17: 31.54$ & $-16: 13: 02.7$ & $13.20 \pm 0.12$ & $10.50 \pm 0.02$ & $9.18 \pm 0.01$ & $\mathrm{OH}-\mathrm{A}$ & 5.0 & 60.6 & \\
\hline \multirow[t]{5}{*}{ g01961-1 } & $18: 24: 50.50$ & $-11: 58: 34.5$ & $>15.5$ & $12.76 \pm 0.07$ & $11.04 \pm 0.03$ & $\mathrm{H}_{2} \mathrm{O}-\mathrm{A}$ & 4.1 & 75.5 & \\
\hline & & & & & & $\mathrm{H}_{2} \mathrm{O}-\mathrm{B}$ & 5.6 & 103.2 & $\mathrm{D}$ \\
\hline & & & & & & $\mathrm{H}_{2} \mathrm{O}-\mathrm{C}$ & 6.8 & 125.3 & $\mathrm{D}$ \\
\hline & & & & & & $\mathrm{H}_{2} \mathrm{O}-\mathrm{D}$ & 9.4 & 173.2 & $\mathrm{D}$ \\
\hline & & & & & & $\mathrm{OH}-\mathrm{A}$ & 3.5 & 64.5 & \\
\hline g02301-1 & $18: 31: 55.82$ & $-09: 03: 03.0$ & $13.70 \pm 0.03$ & $13.73 \pm 0.05$ & $12.99 \pm 0.05$ & $\mathrm{H}_{2} \mathrm{O}-\mathrm{A}$ & 4.2 & 260.6 & \\
\hline & & & & & & $\mathrm{OH}-\mathrm{A}$ & 2.2 & 136.5 & \\
\hline g02343-N & & & $>16.0$ & $>15.1$ & $>15.0$ & $\mathrm{H}_{2} \mathrm{O}-\mathrm{A}$ & - & - & \\
\hline & & & & & & $\mathrm{H}_{2} \mathrm{O}-\mathrm{B}$ & - & - & \\
\hline & & & & & & OH-A & - & - & \\
\hline g02343-1 & $18: 31: 55.65$ & $-08: 33: 52.5$ & $12.40 \pm 0.01$ & $11.80 \pm 0.01$ & $11.39 \pm 0.02$ & $\mathrm{OH}-\mathrm{B}$ & 3.2 & 121.0 & \\
\hline g02478-1 & $18: 33: 30.17$ & $-07: 14: 36.0$ & $16.20 \pm 0.30$ & $14.80 \pm 0.10$ & $13.23 \pm 0.07$ & $\mathrm{H}_{2} \mathrm{O}-\mathrm{A}$ & 8.2 & 306.1 & $\mathrm{D}$ \\
\hline & & & & & & OH-A & 6.8 & 253.8 & $\mathrm{D}$ \\
\hline g02478-N & & & $>16.0$ & $>15.1$ & $>15.0$ & $\mathrm{H}_{2} \mathrm{O}-\mathrm{B}$ & - & - & \\
\hline
\end{tabular}



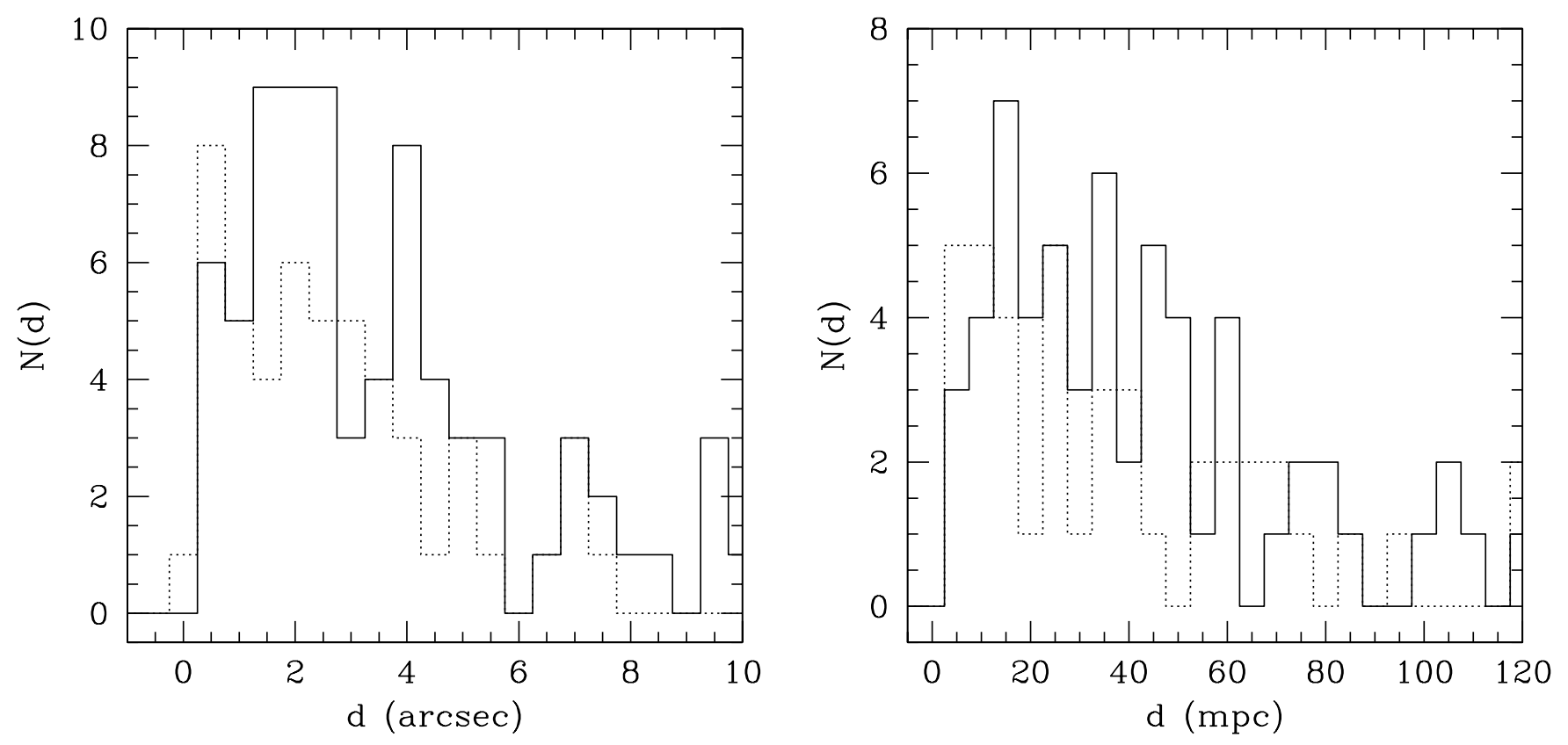

Fig. 2. Distribution of separations between NIR sources and maser components. Continuous line: $\mathrm{H}_{2} \mathrm{O}$ maser components; dotted line: $\mathrm{OH}$ masers components. On the left: observed projected separation on the sky in arcsecond; on the right: projected separation on the plane of the sky in milliparsecs. The distances quoted in Table 1 have been used
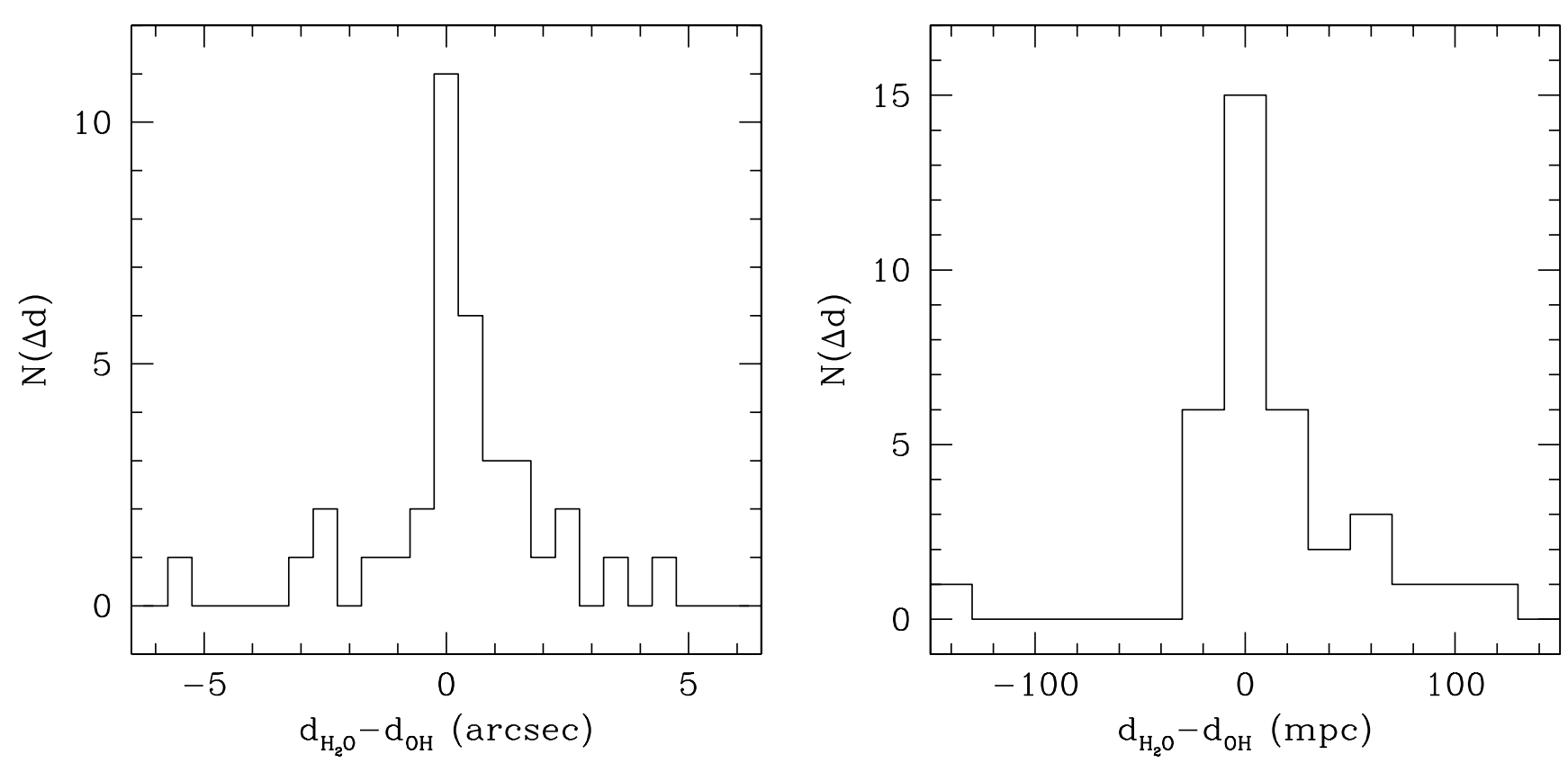

Fig. 3. Distribution of the difference of the separations between the NIR source and the $\mathrm{H}_{2} \mathrm{O}$ and OH maser components. On the left: separation difference in arcsecond; on the right: in milliparsecs 

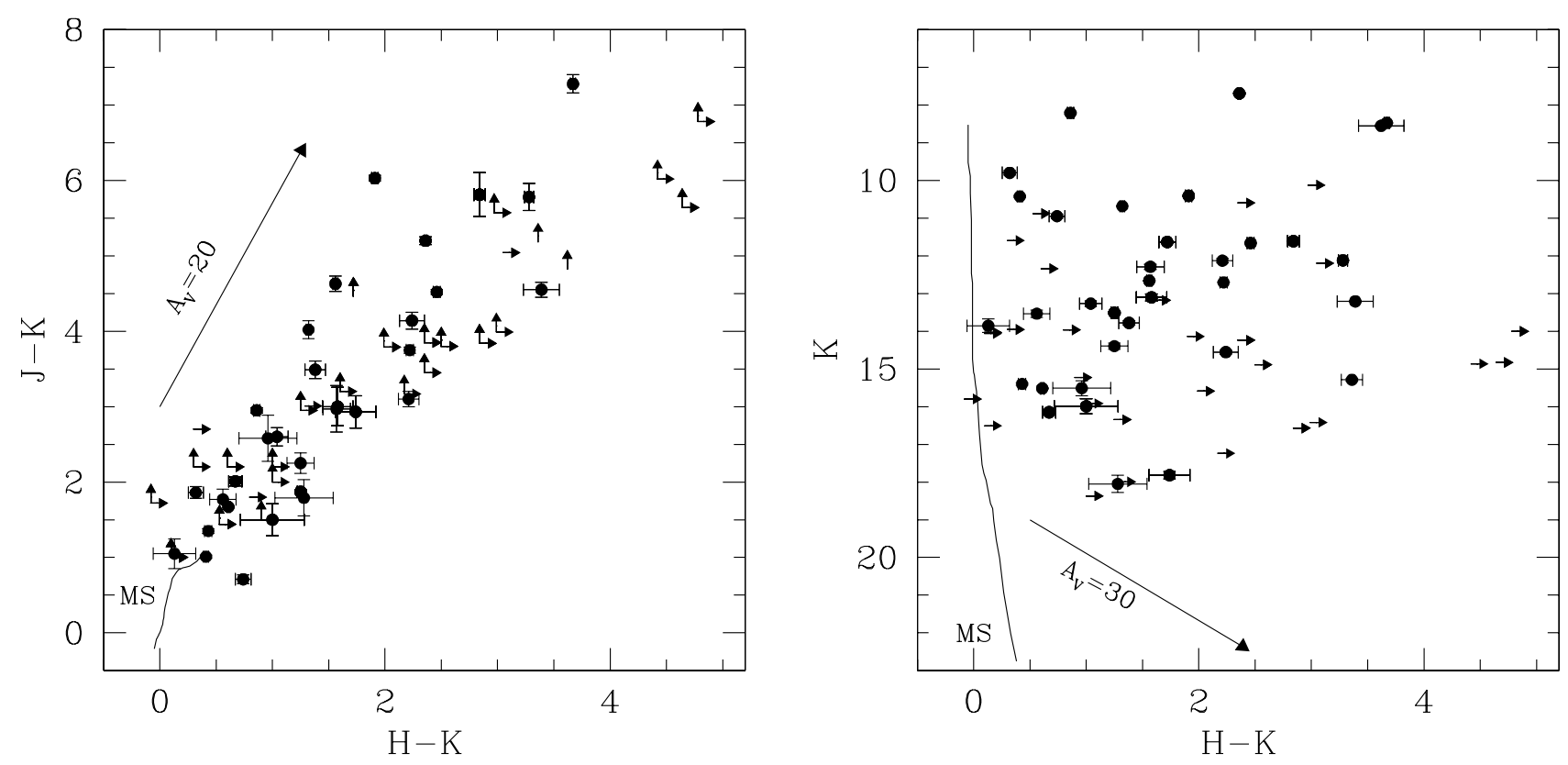

Fig. 4. Colour-colour $(H-K, J-K)$ and colour-magnitude $(H-K, K)$ diagrams for the sources associated with the maser components. In the colour-colour diagram (left) the continuous line represents the colours of Main Sequence stars (from Koornneef 1983), the reddening vector for 20 mag of extinction in the $V$-band has been plotted assuming a Rieke \& Lebofski (1985) exctinction curve. In the colour-magnitude diagram (right) the $K$ magnitudes have been scaled to a distance of $5 \mathrm{kpc}$ without correction for interstellar reddening. The continuous line marks the locus of main sequence stars (Koornneef 1983 and Schmidt-Kaler 1981)
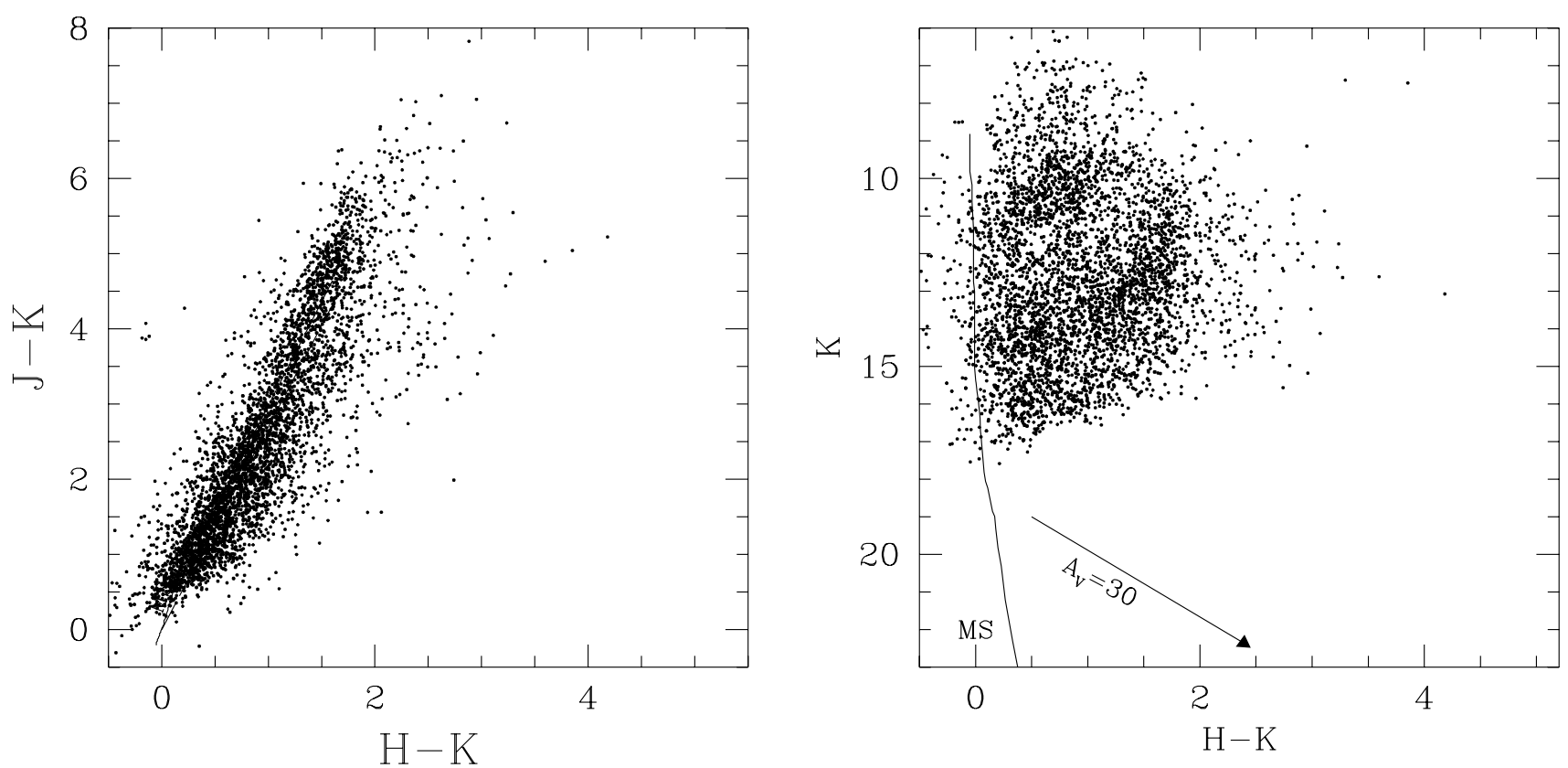

Fig. 5. Colour-colour $(H-K, J-K)$ and colour-magnitude diagrams $(H-K, K)$ for all the sources detected within 1 arcmin from the $\mathrm{H}_{2} \mathrm{O}$ maser reference in the 1994 data. The distance of the field stars is not known, of course, but, in order to compare with Fig. 4, we have scaled the $K$ magnitude to $5 \mathrm{kpc}$ using the maser distance 
Table 2. b) same as Table 2a but for the 1994 observations

\begin{tabular}{|c|c|c|c|c|c|c|c|c|c|}
\hline Name & $\alpha(1950)$ & $\delta(1950)$ & $m_{J}$ & $m_{H}$ & $m_{K}$ & Maser & $d\left({ }^{\prime \prime}\right)$ & $d(\mathrm{mpc})$ & \\
\hline$\overline{\text { g34501-1 }}$ & $16: 53: 17.26$ & $-40: 09: 19.7$ & $>19.3$ & $>18.3$ & $16.13 \pm 0.11$ & $\mathrm{H}_{2} \mathrm{O}-\mathrm{A}$ & 3.5 & 50.9 & \\
\hline g34501-2 & $16: 53: 18.91$ & $-40: 09: 28.3$ & $>19.3$ & $>18.3$ & $15.31 \pm 0.03$ & $\mathrm{H}_{2} \mathrm{O}-\mathrm{B}$ & 1.8 & 26.2 & \\
\hline g34501-3 & $16: 53: 19.72$ & $-40: 09: 38.6$ & $15.64 \pm 0.01$ & $14.72 \pm 0.01$ & $14.29 \pm 0.03$ & $\mathrm{H}_{2} \mathrm{O}-\mathrm{C}$ & 2.7 & 39.3 & \\
\hline \multirow[t]{3}{*}{ g34501-4 } & $16: 53: 19.63$ & $-40: 09: 45.7$ & $>19.3$ & $>18.3$ & $15.46 \pm 0.08$ & $\mathrm{OH}-\mathrm{A}$ & 2.4 & 34.9 & \\
\hline & & & & & & $\mathrm{OH}-\mathrm{B}$ & 1.1 & 16.0 & \\
\hline & & & & & & $\mathrm{OH}-\mathrm{C}$ & 0.2 & 2.9 & \\
\hline \multirow[t]{4}{*}{ g34313-1 } & $16: 54: 43.79$ & $-42: 47: 35.0$ & $17.19 \pm 0.03$ & $15.85 \pm 0.03$ & $15.18 \pm 0.05$ & $\mathrm{H}_{2} \mathrm{O}-\mathrm{A}$ & 1.6 & 24.8 & \\
\hline & & & & & & $\mathrm{H}_{2} \mathrm{O}-\mathrm{B}$ & 1.3 & 20.2 & \\
\hline & & & & & & $\mathrm{H}_{2} \mathrm{O}-\mathrm{C}$ & 3.1 & 48.1 & \\
\hline & & & & & & $\mathrm{OH}-\mathrm{A}$ & 0.5 & 7.8 & \\
\hline g34551-1 & $17: 00: 53.52$ & $-40: 40: 17.2$ & $19.12 \pm 0.13$ & $>17.4$ & $16.11 \pm 0.07$ & $\mathrm{H}_{2} \mathrm{O}-\mathrm{A}$ & 1.3 & 13.2 & \\
\hline g34551-2 & $17: 00: 53.42$ & $-40: 40: 14.8$ & $>19.0$ & $>17.4$ & $12.98 \pm 0.03$ & $\mathrm{OH}-\mathrm{A}$ & 0.6 & 6.1 & \\
\hline g34500-1 & $17: 01: 39.74$ & $-41: 24: 58.4$ & $>19.5$ & $17.68 \pm 0.09$ & $14.32 \pm 0.03$ & $\mathrm{H}_{2} \mathrm{O}-\mathrm{A}$ & 3.9 & 60.5 & \\
\hline \multirow[t]{2}{*}{ g34500-2 } & $17: 01: 40.19$ & $-41: 24: 58.5$ & $>19.5$ & $>18.5$ & $13.86 \pm 0.01$ & $\mathrm{H}_{2} \mathrm{O}-\mathrm{B}$ & 0.6 & 9.3 & \\
\hline & & & & & & $\mathrm{OH}-\mathrm{A}$ & 2.2 & 34.1 & \\
\hline g34541-1 & $17: 06: 02.28$ & $-41: 32: 06.5$ & $14.96 \pm 0.11$ & $13.96 \pm 0.09$ & $12.71 \pm 0.08$ & $\mathrm{H}_{2} \mathrm{O}-\mathrm{A}$ & 1.2 & 13.4 & \\
\hline g34541-2 & $17: 06: 03.92$ & $-41: 32: 10.1$ & $14.77 \pm 0.04$ & $13.24 \pm 0.03$ & $11.02 \pm 0.01$ & OH-A & 0.9 & 10.0 & \\
\hline \multirow[t]{2}{*}{ g34907-1 } & $17: 13: 25.59$ & $-38: 01: 58.3$ & $13.69 \pm 0.01$ & $12.63 \pm 0.01$ & $12.02 \pm 0.01$ & $\mathrm{H}_{2} \mathrm{O}-\mathrm{A}$ & 0.7 & 3.4 & $\mathrm{C}$ \\
\hline & & & & & & $\mathrm{OH}-\mathrm{A}$ & 0.8 & 3.9 & $\mathrm{C}$ \\
\hline \multirow[t]{2}{*}{ g35002-1 } & $17: 14: 22.17$ & $-37: 00: 02.6$ & $17.76 \pm 0.10$ & $16.60 \pm 0.16$ & $13.21 \pm 0.01$ & $\mathrm{H}_{2} \mathrm{O}-\mathrm{A}$ & 0.8 & 19.4 & \\
\hline & & & & & & $\mathrm{OH}-\mathrm{A}$ & 0.3 & 7.3 & \\
\hline g34870-1 & $17: 16: 38.96$ & $-38: 54: 16.8$ & $17.62 \pm 0.07$ & $17.11 \pm 0.12$ & $15.83 \pm 0.23$ & $\mathrm{H}_{2} \mathrm{O}-\mathrm{A}$ & 3.7 & 32.3 & \\
\hline \multirow[t]{4}{*}{ g34870-2 } & $17: 16: 39.76$ & $-38: 54: 12.8$ & $18.53 \pm 0.19$ & $17.34 \pm 0.15$ & $15.60 \pm 0.10$ & $\mathrm{H}_{2} \mathrm{O}-\mathrm{B}$ & 4.2 & 36.7 & \\
\hline & & & & & & $\mathrm{H}_{2} \mathrm{O}-\mathrm{C}$ & 2.0 & 17.5 & \\
\hline & & & & & & $\mathrm{H}_{2} \mathrm{O}-\mathrm{D}$ & 2.0 & 17.5 & \\
\hline & & & & & & $\mathrm{H}_{2} \mathrm{O}-\mathrm{E}$ & 1.2 & 10.5 & \\
\hline \multirow[t]{2}{*}{ g35158-1 } & $17: 22: 02.81$ & $-36: 10: 06.3$ & $>19.0$ & $>17.2$ & $17.28 \pm 0.25$ & $\mathrm{H}_{2} \mathrm{O}-\mathrm{A}$ & 2.5 & 120.0 & \\
\hline & & & & & & $\mathrm{OH}-\mathrm{A}$ & 2.5 & 120.0 & \\
\hline \multirow[t]{3}{*}{ g35158-2 } & $17: 22: 03.37$ & $-36: 10: 08.3$ & $13.80 \pm 0.02$ & $12.89 \pm 0.04$ & $12.36 \pm 0.03$ & $\mathrm{H}_{2} \mathrm{O}-\mathrm{B}$ & 3.0 & 144.0 & \\
\hline & & & & & & $\mathrm{OH}-\mathrm{B}$ & 3.2 & 153.6 & \\
\hline & & & & & & $\mathrm{OH}-\mathrm{C}$ & 2.8 & 134.4 & \\
\hline \multirow[t]{2}{*}{ g35252-1 } & $17: 23: 50.80$ & $-35: 17: 02.0$ & $18.29 \pm 0.10$ & $16.18 \pm 0.07$ & $14.80 \pm 0.06$ & $\mathrm{H}_{2} \mathrm{O}-\mathrm{A}$ & 1.3 & 50.4 & $\mathrm{C}$ \\
\hline & & & & & & $\mathrm{OH}-\mathrm{A}$ & 0.7 & 27.1 & $\mathrm{C}$ \\
\hline \multirow[t]{2}{*}{ g35346-1 } & $17: 23: 33.00$ & $-34: 05: 53.8$ & $17.31 \pm 0.03$ & $15.25 \pm 0.03$ & $12.79 \pm 0.01$ & $\mathrm{H}_{2} \mathrm{O}-\mathrm{A}$ & 0.8 & 32.6 & \\
\hline & & & & & & $\mathrm{OH}-\mathrm{A}$ & 0.6 & 24.4 & \\
\hline \multirow[t]{2}{*}{ g35461-1 } & $17: 27: 00.29$ & $-33: 11: 37.3$ & $>19.0$ & $>17.2$ & $15.21 \pm 0.04$ & $\mathrm{H}_{2} \mathrm{O}-\mathrm{A}$ & 3.9 & 79.4 & \\
\hline & & & & & & $\mathrm{OH}-\mathrm{A}$ & 3.8 & 77.4 & \\
\hline \multirow[t]{3}{*}{ g35914-1 } & $17: 40: 13.99$ & $-29: 37: 57.5$ & $16.44 \pm 0.03$ & $12.32 \pm 0.02$ & $10.41 \pm 0.02$ & $\mathrm{H}_{2} \mathrm{O}-\mathrm{A}$ & 0.6 & 14.5 & $\mathrm{C}$ \\
\hline & & & & & & $\mathrm{H}_{2} \mathrm{O}-\mathrm{B}$ & 1.8 & 43.6 & $\mathrm{C}$ \\
\hline & & & & & & $\mathrm{OH}-\mathrm{A}$ & 0.4 & 9.7 & $\mathrm{C}$ \\
\hline \multirow[t]{2}{*}{ g35997-1 } & $17: 44: 09.18$ & $-29: 10: 57.3$ & $17.26 \pm 0.12$ & $13.65 \pm 0.02$ & $9.98 \pm 0.01$ & $\mathrm{H}_{2} \mathrm{O}-\mathrm{A}$ & 0.7 & 33.9 & \\
\hline & & & & & & $\mathrm{OH}-\mathrm{A}$ & 0.9 & 43.6 & \\
\hline \multirow[t]{2}{*}{ g00214-1 } & $17: 47: 28.29$ & $-27: 05: 01.1$ & $18.49 \pm 0.12$ & $>16.5$ & $13.45 \pm 0.02$ & $\mathrm{H}_{2} \mathrm{O}-\mathrm{A}$ & 2.3 & 99.2 & \\
\hline & & & & & & OH-A & 2.0 & 86.3 & \\
\hline \multirow[t]{2}{*}{ g03520-1 } & $18: 59: 13.06$ & $+01: 09: 11.2$ & $>19.6$ & $>17.6$ & $12.82 \pm 0.01$ & $\mathrm{H}_{2} \mathrm{O}-\mathrm{A}$ & 1.0 & 14.1 & \\
\hline & & & & & & OH-A & 1.7 & 23.9 & \\
\hline
\end{tabular}

within $5^{\prime \prime}$. Hence we do not have strong statistical support for the uniqueness of the association between the maser components and the NIR sources based only on positional coincidence. In fact, due to the high densities of sources we expect some contamination from background/foreground sources. The fields in which the mean separation between the $K$-band sources is much smaller than $5^{\prime \prime}$ have been marked in Table 2 with a "C" in the last column (note that in all those cases the distance between the maser components and the NIR sources is $\lesssim 2^{\prime \prime}$ ).

In order to find additional criteria to establish a physical association we have examined the NIR colours of the close coincident sources and those of background sources.

Since many of the sources coincident with the maser components are not detected at $J$ and $H$, we will use the 
$(H-K, J-K)$ colour-colour diagram to investigate the nature of the infrared sources, as in Paper I.

Since there is no systematic difference in the colour characteristics of the NIR sources associated to the $\mathrm{H}_{2} \mathrm{O}$ and $\mathrm{OH}$ masers (in fact in many cases both masers are associated with the same NIR source), we will discuss the nature of the sources associated with both masers together.

In Fig. 4 the $(H-K, J-K)$ colour-colour diagram and the $(H-K, K)$ colour magnitude diagram for the sources coincident with the maser components are presented. The colours of main sequence (MS) stars are from Koornneef (1983), the magnitudes from Schmidt-Kaler (1981) and the reddening vectors have been plotted assuming a Rieke \& Lebofski (1985) extinction law. The $K$ magnitudes of all the sources have been normalized to a distance of $5 \mathrm{kpc}$ from the Sun, assuming the distances quoted in Table 1. In Fig. 5 similar plots are given for the sources detected within 1 arcmin from the maser reference of FC89, presumably field stars.

From a comparison of Figs. 4 and 5 it can be clearly seen that the field stars are distributed close with the locus of main sequence and reddened main sequence stars, while almost all the sources coincident with the maser components show a conspicuous infrared excess.

Consequently, independent support to a physical association between maser components and NIR sources is given by the fact that the NIR sources close coincident with the maser components show NIR excesses, while the field stars are located along the reddening line.

This provides proof of a distinct property of the maser associated sources. As discussed in Paper I and in Testi et al. (1997) the NIR excess emission is probably the result of the superposition of (at least) three main contributions: i) the heavily extincted stellar photosphere of a massive young stellar object (the ultimate energy source of the entire system), ii) a contribution from free-free and free-bound continum emission from ionized gas and iii) the emission of the hot dust surrounding the YSO. In particular, in the earliest evolutionary stages, when dust is still present very close to the exciting star, a hot dust shell dominates the emission at wavelengths longward of $\sim 1.5 \mu \mathrm{m}$ and the object is effectively detectable at $K$ band even if still embedded in the parental molecular clump.

Strong support for the association procedure is also provided by the three sources of this sample that have been studied in detail by Persi et al. $(1996,1997)$ and Testi et al. (1997). The arcsecond resolution comparison of the NIR images with mid infrared and/or thermal molecular line emission and mm-wave continuum observations show that the NIR sources associated with the maser emission using the "nearest neighbour" criterion are also associated with high density molecular clumps and dense cool dust clouds, which are other indicators of the presence of a YSO. Due to the absence of a UCHII region (in spite of the high luminosities inferred from the IRAS far infrared observations), the three NIR sources discussed above are believed to be in a very early phase, when the size of the ionized region around the young star is so small that the radio continuum emission is strongly self-absorbed. Consequently, the UC HII region is undetectable at radio wavelengths (see also the discussion in Testi et al. 1997), while hot dust emission can be detected even through strong absorption.

In a forthcoming paper (Testi et al. 1997 in preparation) we will discuss the NIR properties of the sources associated with the masers in the framework of a model which takes into account the various emission components.

\section{Conclusions}

We have presented near infrared observations at $J, H$ and $K$ broad bands of the regions surrounding 31 SFR galactic $\mathrm{H}_{2} \mathrm{O}$ and $\mathrm{OH}$ masers. The main conclusions, taking into account also the results of Paper I, are as follows:

1. we identified a K-band source within $5^{\prime \prime}$ from the $\mathrm{H}_{2} \mathrm{O}$ maser components in $73 \%$ of the cases $(91 \%$ within $\left.10^{\prime \prime}\right)$ and in $88 \%$ in the case of $\mathrm{OH}$ masers (95\% within $\left.10^{\prime \prime}\right)$;

2. the NIR sources close to the masers and which are believed to be physically associated with them, have colours which differs from those of the field stars, showing in most cases a remarkable near infrared excess at $2.2 \mu \mathrm{m}$;

3. the separation between the maser components and the associated NIR sources is in most cases less than $10^{4}$ $\mathrm{AU}$;

4. the NIR sources associated with the maser components appear to be young massive stars in their earliest evolutionary stages.

One of the major results of this work is that even in fields overcrowded by foreground/background stars it is possible to find the NIR source physically associated with the masers by examining the positional coincidence and the NIR colours. We argue that this is the general situation, namely that the masers are always associated with a NIR source with strong infrared excess and that this is the ultimate energy source for the maser action.

Acknowledgements. We thank R. Forster for providing unpublished VLA data. Support from C.N.R.-N.A.T.O. Advanced Fellowship program and from NASA's Origins of Solar Systems program (through grant NAGW-4030) is gratefully aknowledged.

\section{References}

Braz M.A., Epchtein N., 1982, A\&A 111, 91

Casali M., Hawardeen T., 1992, JCMT-UKIRT Newslett. No. 4, p. 33

Cesaroni R., Churchwell E., Hofner P., Walmsley C.M., Kurtz S., 1994, A\&A 288, 903 
Cesaroni R., Felli M., Testi L., Walmsley C.M., Olmi L., 1997, A\&A 325, 725

Codella C., Felli M., Natale V., Palagi F., Palla F., 1994, A\&A 291, 261

Codella C., Testi L., Cesaroni R., 1997, A\&A 325, 282

Elitzur M., Hollenbach D.J., McKee C.F., 1989, ApJ 346, 983

Epchtein N., Lépine J.R.D., 1981, A\&A 99, 210

Evans N.J.II, Beckwith S., Brown R.L., Gilmore W., 1979, ApJ 227,450

Forster J.R., Caswell J.L., 1989, A\&A 213, 339, FC89

Genzel R., Downes D., 1977, A\&AS 30, 145

Hofner P., Churchwell E., 1996, A\&AS 120, 283

Hofner P., Kurtz S., Churchwell E., Walmsley C.M., Cesaroni R., 1996, ApJ 460, 359

Hunt L.K., Testi L., Borelli S., Maiolino R., Moriondo G., 1994 Technical Report 4/94, Arcetri Astrophysical Observatory

Jenness T., Scott P.F., Padman R., 1995, MNRAS 276, 1024

Koornneef J., 1983, A\&A 128, 84

Moorwood A., Salinari P., 1981a, A\&A 94, 299

Moorwood A., Salinari P., 1981b, A\&A 102, 197
Olmi L., Cesaroni R., Neri R., Walmsley C.M., 1996, A\&A 315, 565

Palla F., Cesaroni R., Brand J., et al., 1993, A\&A 280, 509

Persi P., Felli M., Lagage P.O., Roth M., Testi L., 1997, A\&A 327, 299

Persi P., Roth M., Tapia M., et al., 1996, A\&A 307, 591

Persson S.E., West S.C., Carr D.M., Sivaramakrishnan A., Murphy D.C., 1992, PASP 104, 204

Rieke G.H., Lebofsky M.J., 1985, ApJ 288, 618

Schmidt-Kaler Th., 1981, in Landolt-Börnstein, Gruppe VI, Bond 2, 1 (Publ. Springer)

Testi L., 1993, Technical Report 10/93, Arcetri Astrophysical Observatory

Testi L., Felli M., Persi P., Roth M., 1994, A\&A 288, 634, Paper I

Testi L., Felli M., Persi P., Roth M., 1997, A\&A (in press)

Tofani G., Felli M., Taylor G.B., Hunter T.R., 1995, A\&AS 112,299

Wood D.O.S., Churchwell E., 1989, ApJ 340, 265 